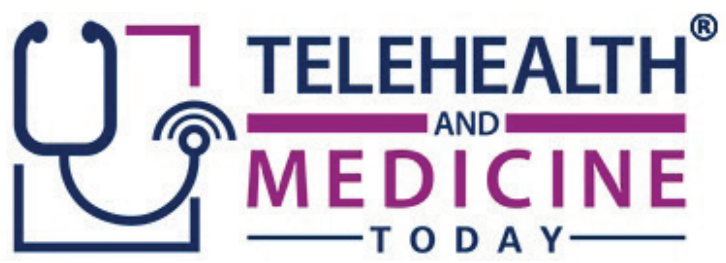

\title{
Prenatal telehealth during the pandemic: sociodemographic and clinical associations
}

Cheng Gao, $\mathrm{PhD}^{1 * \odot}$; Sarah Osmundson, $\mathrm{MD}^{2} \odot$; Bradley A. Malin, $\mathrm{PhD}^{1,3,4} \odot$ and You Chen, $\mathrm{PhD}^{1,4}$ ๑)

Affiliations: ${ }^{1}$ Department of Biomedical Informatics, Vanderbilt University Medical Center, Nashville, TN, USA; ${ }^{2}$ Department of Obstetrics and Gynecology, Vanderbilt University Medical Center, Nashville, TN, USA; ${ }^{3}$ Department of Biostatistics, Vanderbilt University Medical Center, Nashville, TN, USA; ${ }^{4}$ Department of Electrical Engineering \& Computer Science, School of Engineering, Vanderbilt University, Nashville, TN, USA

Corresponding Author: *Cheng Gao, Email: cheng.gao@vumc.org

Keywords: Access Barriers, COVID-19, Health Disparities, Obstetric Care, Perinatal Care, Sociodemographic, Telehealth

Section: Original Research

Objectives: Like in other areas of care affected by the COVID-19 pandemic, telehealth (both audio and video) was rapidly adopted in the obstetric setting also. However, how telehealth was used in obstetric care and whether there are disparities in its use have not been investigated. We performed a retrospective analysis of electronic health records (EHR) data to characterize the sociodemographic and clinical factors associated with telehealth use among patients who received prenatal care.

Materials and methods: The study period covered the period 23 March 2020 to 02 July 2020, during which time 2,521 patients received prenatal care at Vanderbilt University Medical Center, Nashville, Tennessee, a large academic medical center. We applied a generalized logistic regression to measure the relationship between the patients' sociodemographic factors (age, race, ethnicity, urbanization level, and insurance type), pregnancy complications (namely, Type 2 diabetes, chronic hypertension, and fetal growth restriction), and telehealth usage, as documented in the EHR.

Results: There were 2,521 patients with 16,516 prenatal care visits during the study period, and 938 (37.2\%) of the patients participated in at least one of the 1,934 virtual prenatal care visits. Prenatal visits were more likely to be conducted through telehealth for patients who were older than 25 years, and who lived in rural areas. In addition, patients with Type 2 diabetes were more likely than women without health complications to use telehealth in their prenatal care (adjusted odds ratio [aOR] 7.20 [95\% 
confidence interval \{95\% CI\} 4.22-12.85]). By contrast, patients from racial and ethnic minority groups were less likely to have a telehealth encounter than White or non-Hispanic patients (aOR 0.57 [95\% CI 0.44-0.73] and aOR 0.62 [95\% CI 0.44-0.86], respectively). Additionally, patients who were on Medicaid were less likely than other insured groups to use telehealth (aOR 0.55 [95\% CI 0.45-0.68]).

Discussion: There were disparities in telehealth use for prenatal care. Over $45 \%$ of the patients in certain groups (e.g. White, older than 35 years old, and commercially insured) received prenatal care via telehealth at least once, while less than $25 \%$ of other groups (e.g. younger than 25 years and Hispanic) used it. Women who used telehealth significantly less in their prenatal care were Black, other races (e.g. Native American and Native Hawaiian), Hispanic, and on Medicaid. Further work is advised to investigate the reasons for such disparities in the current telehealth setting.

Conclusions: While telehealth expanded prenatal care access for childbearing women during the COVID-19 pandemic, this study suggests that there are statistically significant differences in telehealth use among the patients who utilized such settings.

$\mathrm{T}$ elehealth (both audio and video) is broadly defined as the use of electronic information and telecommunication technologies to support health-related services (1). Historically, telehealth has brought healthcare to patients living in rural areas that lack sufficient access to in-person healthcare services (2). It has further played an important role in supporting healthcare during disasters when travel is limited and in-person care is disrupted $(3,4)$ The range, as well as use, of telehealth services has increased dramatically over the past several decades. It now incorporates a wide variety of services, including long-distance clinical care, patient and professional health-related education, and health administration (1).

The World Health Organization (WHO) declared the 2019 novel coronavirus (COVID-19) a public health emergency of international concern on 30 January 2020 (5). After the WHO officially classified COVID-19 as a pandemic $(5,6)$ the number of infections in the United States (U.S.) grew exponentially (7). Due to rapid viral transmission (8), lack of effective antiviral treatments, and the unknown long-term sequelae of viral infection (9), the Centers for Disease Control and Prevention (CDC) recommended that healthcare providers expand telehealth service to their patients (10). In February 2020, the American College of Obstetrics and Gynecology (ACOG) published a guidance for implementing telehealth, which encouraged its use in routine prenatal care, consultations with specialty services (e.g. psychiatry, endocrinology, and nephrology), and remote reading of ultrasound imaging (11).

Although telehealth has been recommended by the CDC and the ACOG, it should be customized and scaled to meet the healthcare needs of a wide variety of special populations (12). Various healthcare organizations created procedures to determine which obstetrics patients are candidates for telehealth service $(13,14)$ how telehealth should be optimized for these patients $(15,16)$ and which types of prenatal visits can be delivered via virtual care (17) Specific recommendations were made to incorporate telehealth for high-risk pregnancies (e.g. hypertensive disorder, pregestational and gestational diabetes mellitus, and maternal neurologic conditions) (18). In light of these recommendations, telehealth was rapidly 
implemented at various medical centers across the country; however, rapid implementation of telehealth may exacerbate disparities in healthcare access (19-21). This potential was highlighted by a study of 5 weeks of data from Columbia University Irving Medical Center, in which women with Medicaid insurance experienced greater difficulties transitioning to telehealth than those with commercial insurance (17). Similarly, researchers in the Department of Veterans Affairs found that Black veterans had a decreased likelihood of using virtual care during the pandemic (22). Although these studies provided insight into health disparities in telehealth implementation, they did not adjust for sociodemographic factors and health conditions.

In this study, we investigate prenatal care visits during the COVID-19 pandemic at Vanderbilt University Medical Center (VUMC) to assess the utilization of telehealth over time, and its relationship with sociodemographic factors and clinical conditions.

\section{MATERIALS AND METHODS}

The Department of Obstetrics and Gynecology at VUMC provides prenatal care and delivery services to over 4,500 women per year and serves as a regional referral center for both maternal care and fetal anomalies. The Epic electronic health record (EHR) system which serves as the central source of documentation for patient care, documents patients' demographics, their prenatal visits, and health conditions during these visits.

We collected demographics (age, race [White, Black, Asian, other races \{Native American and Native Hawaiian\}], and ethnicity [Hispanic and non-Hispanic]), pre-pregnancy body mass index (BMI), residential county, and types of health insurance associated with each patient's EHR from 23 March to 02 July 2020. Maternal age was categorized into four groups: (1) younger than 18 years, (2) 18-25 years, (3) 26-35 years, and (4) over 35 years. These categories were selected because women younger than 18 and older than 35 years can be considered higher risk pregnancies (23). Pre-pregnancy BMI was also split into four categories: (1) less than or equal to 18.5 (underweight), (2) 18.5-25 (normal weight), 3) 25-30 (overweight), and (4) greater than or equal to 30 (obese). The conversions for these two variables allowed us to model a more complex relationship in a generalized linear model, rather than monotonically increasing or decreasing. In addition, each patient was assigned to an urbanization level based on their residential county according to the six-level urban-rural classification scheme for U.S. counties and county-equivalent entities developed by the National Center for Health Statistics (24). The most urban category consists of 'central' counties of large metropolitan areas (denoted as 1), whereas the most rural category consists of non-metropolitan 'non-central' counties (denoted as 6). Lastly, the insurance type was represented as one of five types: (1) commercial (e.g. Aetna, United Healthcare, or Blue Cross Blue Shield), (2) Medicare, (3) Medicaid, (4) some other forms of governmental insurance, and (5) other.

We used pregnant status (e.g. gestational age) to retrieve all prenatal care visits from the EHR. Each visit included date of service, visit type (telehealth or non-telehealth), and prenatal conditions characterized by ICD-10-CM. Three common prenatal conditions that require frequent patient monitoring are associated with complex pregnancies: (1) Type 2 diabetes, (2) chronic hypertension, and (3) fetal growth restriction.

To compare telehealth use between patient subpopulations, we applied a generalized logistic regression in which the dependent variable is a 
binary indicator of telehealth use (i.e. use of telehealth at any point during the time period), and the independent variables were demographics, BMI, urbanization level, insurance type, and the prenatal complication conditions. We report the adjusted odds ratio (aOR) with a $95 \%$ confidence interval (CI) and Bonferroni corrected $p$-value.

\section{RESULTS}

This section begins with telehealth use for the studied population during the pandemic. We then report a summary of the sociodemographic and clinical factors with respect to telehealth use. This section concludes with an analysis of the relationship between the aforementioned factors and telehealth use.

\section{Telehealth use in prenatal care}

Figure 1 shows the number of prenatal visits that occurred between 23 March 2020 and 02 July 2020. There were between 200 and 300 prenatal encounters per day. Telehealth and in-person visits are shown in blue and orange, respectively. Telehealth visits rapidly escalated in the week of 23 March, peaked during the week of 07 April, and deescalated slowly after the week of 29 June.
This reflects that telehealth was suggested for obstetric patients through the early summer as the COVID-19 outbreak in Tennessee grew. As such, we focused our study on 23 March to 02 July.

\section{Sociodemographic and clinical factors}

Table 1 summarizes the sociodemographic and clinical factors of 2,521 women who received prenatal care within the dataset; 938 (37.2\%) of these patients had at least one telehealth visit in their prenatal care. There were 1,934 telehealth visits, with an average of 2.1 prenatal telehealth encounters per patient.

There are several notable observations regarding differences in telehealth utilization. First, we consider how the age of the patient influences its use. Younger women were less likely to use telehealth: over $40 \%$ of the older population used telehealth, yet only $13 \%$ of women younger than 18 years and $23 \%$ of women aged $18-25$, used telehealth in their prenatal care. Second, we observed a lower amount of telehealth use by racial and ethnic minorities: only $29 \%$ of Black women had telehealth visits, compared to $44 \%$ of White patients. Similarly, 19\% of Hispanic

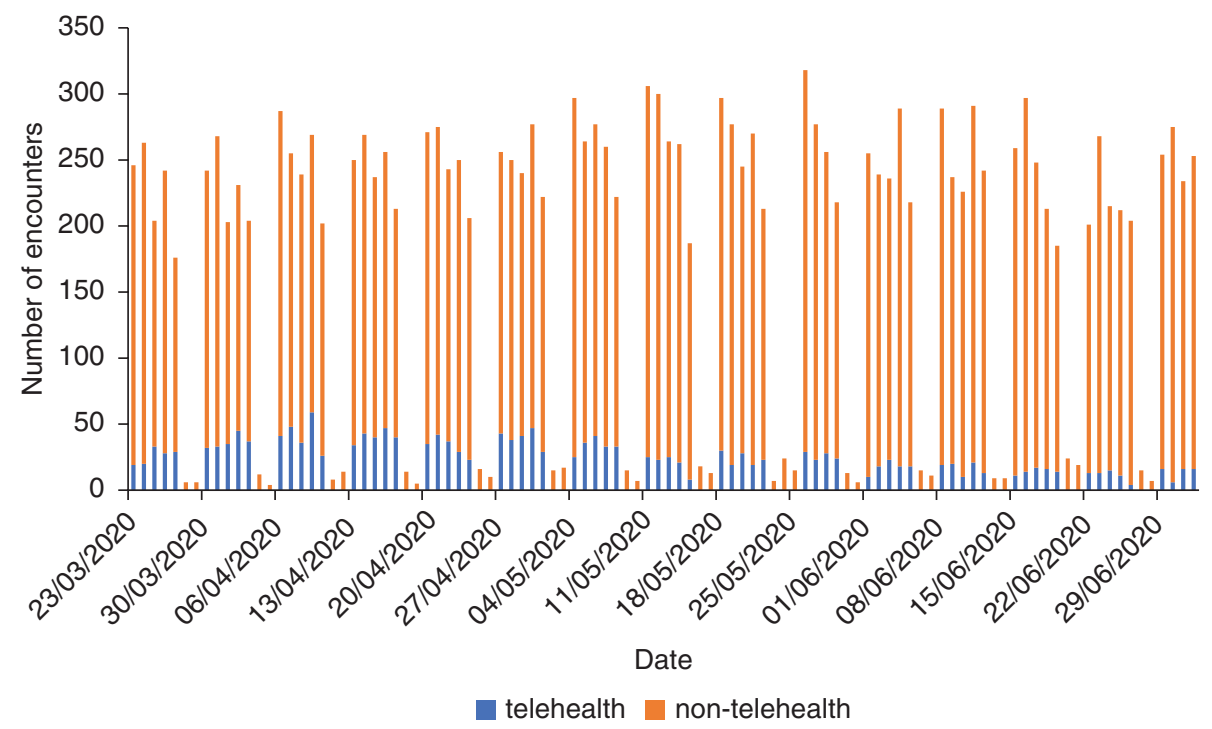

Figure 1. The number of prenatal encounters by visit type (telehealth vs. non-telehealth) over each day of the study period (15 weeks: 23 March to 02 July 2020). 
Table 1. Characteristics of the study population (2,521 patients) and telehealth use between 23 March and 02 July 2020

\begin{tabular}{|c|c|c|c|c|}
\hline Variable & Category & Patients & $\begin{array}{l}\text { Patients with at least } \\
\text { one telehealth } \\
\text { encounter }\end{array}$ & $\begin{array}{l}\text { Patients lacking a } \\
\text { telehealth encounter }\end{array}$ \\
\hline \multirow{4}{*}{ Age } & $<18$ years & 36 & $4(11.11 \%)$ & $32(88.89 \%)$ \\
\hline & $18-25$ years & 647 & $154(23.80 \%)$ & $493(76.20 \%)$ \\
\hline & $26-35$ years & 1452 & $594(40.91 \%)$ & $858(59.09 \%)$ \\
\hline & $>35$ years & 386 & $186(48.19 \%)$ & $200(51.81 \%)$ \\
\hline \multirow{3}{*}{ Ethnicity } & Non-Hispanic & 2098 & $853(40.66 \%)$ & $1245(59.34 \%)$ \\
\hline & Hispanic & 376 & $71(18.88 \%)$ & $305(81.12 \%)$ \\
\hline & Undecided & 47 & $14(29.79 \%)$ & $33(70.21 \%)$ \\
\hline \multirow{4}{*}{ Race } & White & 1551 & $683(44.04 \%)$ & $868(55.96 \%)$ \\
\hline & Black & 442 & $132(29.86 \%)$ & $310(70.14 \%)$ \\
\hline & Asian & 126 & $44(34.92 \%)$ & $82(65.08 \%)$ \\
\hline & Other & 402 & $79(19.65 \%)$ & $323(80.35 \%)$ \\
\hline \multirow{6}{*}{$\begin{array}{l}\text { Urbanization } \\
\text { level }\end{array}$} & 1 (the most urban) & 1313 & $415(31.61 \%)$ & $898(68.39 \%)$ \\
\hline & 2 & 884 & $337(38.12 \%)$ & $547(61.88 \%)$ \\
\hline & 3 & 201 & $113(56.22 \%)$ & $88(43.78 \%)$ \\
\hline & 4 & 18 & $12(66.67 \%)$ & $6(33.33 \%)$ \\
\hline & 5 & 65 & $38(58.46 \%)$ & $27(41.54 \%)$ \\
\hline & 6 (the most rural) & 40 & $23(57.5 \%)$ & $17(42.5 \%)$ \\
\hline \multirow{4}{*}{ BMI } & $\leq 18.5$ & 37 & $9(24.32 \%)$ & $28(75.68 \%)$ \\
\hline & $18.6-24.9$ & 846 & $329(38.89 \%)$ & $517(61.11 \%)$ \\
\hline & $25-30$ & 730 & $244(33.42 \%)$ & $486(66.58 \%)$ \\
\hline & $\geq 30.1$ & 908 & $356(39.21 \%)$ & $552(60.79 \%)$ \\
\hline \multirow{3}{*}{$\begin{array}{l}\text { Prenatal } \\
\text { conditions }\end{array}$} & $\begin{array}{l}\text { Type } 2 \text { diabetes } \\
\left(\mathrm{O} 24.1^{*}, \mathrm{O} 24.3^{*},\right. \\
\left.\mathrm{O} 24.8^{*}\right)\end{array}$ & 91 & $72(79.12 \%)$ & $19(20.89 \%)$ \\
\hline & $\begin{array}{l}\text { Chronic hypertension } \\
\left(010 .^{*}, \mathrm{I} 10 .^{*}, \mathrm{I} 12 .^{*},\right. \\
\left.\mathrm{I13} .^{*}, \mathrm{I} 15 .^{*}\right)\end{array}$ & 187 & $96(51.34 \%)$ & $91(48.66 \%)$ \\
\hline & $\begin{array}{l}\text { Fetal growth } \\
\text { restriction }\left(\mathrm{O} 36.5^{*}\right)\end{array}$ & 219 & $89(40.64 \%)$ & $130(59.36 \%)$ \\
\hline \multirow{5}{*}{ Insurance type } & Commercial & 1141 & $552(48.38 \%)$ & $589(51.62 \%)$ \\
\hline & Medicaid & 899 & $261(29.03 \%)$ & $638(70.97 \%)$ \\
\hline & Other governmental & 402 & $89(22.14 \%)$ & $313(77.86 \%)$ \\
\hline & Other & 59 & $24(40.68 \%)$ & $35(59.32 \%)$ \\
\hline & Medicare & 20 & $12(60.00 \%)$ & $8(40.00 \%)$ \\
\hline
\end{tabular}

* refers to the ICD code for that disease. 
women used telehealth, compared to $41 \%$ of non-Hispanic patients.

Third, urbanization levels were also associated with differences in telehealth. Patients who lived in large central and fringe metropolitan areas (urbanization levels 1 and 2) were less likely to use telehealth than those who lived in rural areas (urbanization levels 3 through 6). Over half of the patients who lived in rural areas had telehealth prenatal visits.

Fourth, patients with Type 2 diabetes were more likely to use telehealth than women without medical complications $(79.1 \%$ vs. $35.6 \%)$. Finally, we found that health insurance types affected telehealth use. Women who were on Medicaid and other governmental insurance programs (e.g. Tricare) were less likely to use telehealth than those who were commercially insured: Medicaid 29\%; other governmental programs $22 \%$; and commercial insurance $48 \%$.

\section{Differential telehealth usage by} sociodemographic and clinical factors Table 2 illustrates the differences in telehealth use with respect to sociodemographic and clinical factors. There are several findings worth

Table 2. Adjusted Model: Characteristics of the study population associated with telehealth use

\begin{tabular}{|c|c|c|c|}
\hline Variable & Category & $\begin{array}{l}\text { Odds ratio ( } 95 \% \\
\text { confidence interval) }\end{array}$ & $p$ \\
\hline \multirow{3}{*}{$\begin{array}{l}\text { Age } \\
\text { (Reference: 18-25) }\end{array}$} & $<18$ & $0.559(0.153-1.546)$ & 0.3128 \\
\hline & $26-35$ & $2.061(1.637-2.607)$ & $<0.0001 *$ \\
\hline & $>35$ & $2.836(2.108-3.825)$ & $<0.0001 *$ \\
\hline \multirow{2}{*}{$\begin{array}{l}\text { Ethnicity (Reference: } \\
\text { non-Hispanic) }\end{array}$} & Hispanic & $0.617(0.438-0.864)$ & 0.0053 \\
\hline & Undecided & $1.040(0.511-2.020)$ & 0.9093 \\
\hline \multirow{3}{*}{$\begin{array}{l}\text { Race } \\
\text { (Reference: White) }\end{array}$} & Black & $0.568(0.437-0.734)$ & $<0.0001^{*}$ \\
\hline & Asian & $0.727(0.484-1.081)$ & 0.1196 \\
\hline & Other & $0.564(0.412-0.766)$ & $0.0003 *$ \\
\hline \multirow{5}{*}{$\begin{array}{l}\text { Urbanization level } \\
\text { (Reference: 1) }\end{array}$} & 2 & $1.013(0.831-1.234)$ & 0.8988 \\
\hline & 3 & $2.710(1.937-3.802)$ & $<0.0001^{*}$ \\
\hline & 4 & $3.936(1.439-11.897)$ & 0.0097 \\
\hline & 5 & $2.500(1.458-4.343)$ & $<0.0001 *$ \\
\hline & 6 & $2.478(1.236-5.033)$ & 0.0108 \\
\hline \multirow{3}{*}{ Prenatal conditions } & Type 2 Diabetes & $7.199(4.217-12.845)$ & $<0.0001 *$ \\
\hline & Chronic hypertension & $1.329(0.946-1.865)$ & 0.1001 \\
\hline & Fetal growth restriction & $1.236(0.903-1.686)$ & 0.1823 \\
\hline \multirow{3}{*}{$\begin{array}{l}\text { BMI } \\
\text { (Reference: 18.5-25 }\end{array}$} & $<18.5$ & $0.510(0.215-1.107)$ & 0.1037 \\
\hline & $25-30$ & $0.828(0.660-1.036)$ & 0.0995 \\
\hline & $>30$ & $0.907(0.729-1.129)$ & 0.3841 \\
\hline \multirow{4}{*}{$\begin{array}{l}\text { Insurance (Reference: } \\
\text { Commercial) }\end{array}$} & Medicaid & $0.550(0.449-0.675)$ & $<0.0001 *$ \\
\hline & Other Governmental & $0.410(0.298-0.559)$ & $<0.0001 *$ \\
\hline & Other & $0.724(0.414-1.250)$ & 0.2524 \\
\hline & Medicare & $1.653(0.609-4.673)$ & 0.3275 \\
\hline
\end{tabular}

* indicates significance at the 0.05 level after Bonferroni correction. 
highlighting. First, age influenced telehealth use. Women over 25 years old (26-35 and >35) were more likely to use telehealth in their prenatal care compared to younger women $(\mathrm{aOR}[95 \% \mathrm{CI}]$ : 2.06 [1.64-2.61] and 2.84 [2.11-3.83], respectively). Second, racial and Hispanic minorities used telehealth less than White women. Specifically, women who were Black or of other races were substantially less likely to have telehealth visits than White women (aOR [95\% CI]: 0.57 [0.44-0.73] and 0.56 [0.41-0.77], respectively). Similarly, Hispanics used telehealth less than non-Hispanics (aOR [95\% CI]: 0.62 [0.44-0.86]). Third, a difference in telehealth use existed among the six urbanization levels. Telehealth services were more likely to be utilized by pregnant women who lived in rural areas (urbanization level [aOR $\{95 \% \mathrm{CI}\}$ ]: 3 [2.71 \{1.94-3.80\}]; 5 [2.50\{1.46-4.34\}]; 6 [2.48 $\{1.24-5.03\}])$ than those who lived in large metropolitan areas. Fourth, telehealth use was associated with pregnancy complications.

Women with Type 2 diabetes were more likely to have telehealth services (aOR [95\% CI]:7.20 [4.22-12.85]) than women without complications. Finally, government-assisted insurance was related to less telehealth usage. Women with Medicaid and other governmental insurances were much less likely $(\mathrm{aOR}[95 \% \mathrm{CI}]$ : 0.55 [0.45-0.68] and 0.41 [0.30-0.56], respectively) to receive prenatal care via telehealth than those who were commercially insured.

\section{DISCUSSION}

This study evaluated telehealth use for obstetric patients in their prenatal care at a large academic hospital during the COVID-19 pandemic. The findings indicate that telehealth was rapidly deployed for prenatal care over the first several months of the pandemic. We also observed that there were differences among the patients who used telehealth in this population. Notably, telehealth was more likely to be used by older patients, those who lived in rural areas, or those who exhibited certain clinical complications (e.g. Type 2 diabetes).

Our findings have several notable implications in the expansion of telehealth for policymakers in the post-COVID-19 era. First, while we cannot know why some patients use telehealth more than others, barriers to access are likely a factor. For example, Hispanic patients may be reluctant to use telehealth due to low English proficiency or a current telehealth service without clinicians who speak their language. A previous study showed that limited English proficiency was associated with a lower rate of telehealth use (25). Besides language barriers, Medicaidinsured patients may not be able to afford a reliable internet connection and proper devices for video-conferencing since most are in lowincome households (26), even though Medicaid has covered telehealth services since the start of the pandemic (27).

Second, women living in large metropolitan areas underutilize telehealth services. The probable reason for this is that women living within a short distance to the medical center prefer to see doctors in person, and not using telehealth. These women represent the largest proportion of obstetric patients, yet less than one-third used telehealth during their prenatal care. In contrast, over half of the patients who live in rural areas used telehealth. The urban population may represent an opportunity for telehealth expansion.

Despite these notable findings and implications, there are several limitations to this study. First, this investigation only reflects telehealth use in one healthcare setting, a medical center that provides prenatal care for many high-risk pregnancy patients in nearby areas, so the 
findings may not generalize for other healthcare systems. Second, although our analysis indicates that certain subpopulations were less likely to use telehealth, the reasons (or barriers) for not using telehealth are not fully known from this study design. Additional social determinants of health need to be incorporated in further studies, including years of education, lifestyle choice, and poverty level. Third, this study focused on the telehealth visit itself, and did not consider patients' willingness to use this new technology. Surveys should be distributed to patients regarding the quality and comprehensiveness of telehealth visits, which facilitates further implementation of telehealth.

\section{CONCLUSIONS}

This study showed that telehealth was rapidly adopted for prenatal care during the COVID-19 pandemic at a large academic medical center. Our findings indicated that younger women, minority races and ethnicities, those living in metropolitan areas, and those insured with Medicaid had significantly lower use of telehealth services. In addition, women with Type 2 diabetes had more telehealth use than those without diabetes. We believe that further investigation into the reasons for such differential use, as well as potential solutions to more equitably dispensed healthcare services, is warranted.

Conflict of Interest and Funding: The authors have no competing interests to declare. This research was supported, in part, by Academic Research Program at Vanderbilt University Medical Center.

Contribution: $\mathrm{CG}, \mathrm{SO}, \mathrm{BM}$, and $\mathrm{YC}$ conceived the presented idea and performed the data collection and analysis, method and metric design and development, experiment design, evaluation and interpretation of the experiments, and writing and revision of the manuscript.

\section{REFERENCES}

1. Health Resources and Services Administration (HRSA). Telehealth programs [Internet]. [cited 05 October 2021]. Available from: https://www.hrsa. gov/rural-health/telehealth/index.html

2. Lurie N, Carr BG. The role of telehealth in the medical response to disasters. JAMA Int Med. 2018;178(6):745-6. https://doi.org/10.1001/ jamainternmed.2018.1314

3. Der-Martirosian C, Chu K, Dobalian A. Use of telehealth to improve access to care at the U.S. Department of Veterans Affairs during the 2017 Atlantic hurricane season. Disaster Med Public Health Prep. 2020;1-5. https://doi. org/10.1017/dmp.2020.88

4. Der-Martirosian C, Heyworth L, Chu K, Mudoh Y, Dobalian A. Patient characteristics of VA telehealth users during Hurricane Harvey. $J$ Prim Care Community Heal. 2020;11:2150132720931715. https://doi. org/10.1177/2150132720931715

5. World Health Organization (WHO. Emergency Committee regarding the outbreak of novel coronavirus (2019nCoV) [Internet]. [cited 05 October 2021]. Available from: https:// www.who.int/docs/default-source/ coronaviruse/transcripts/ihr-emergencycommittee-for-pneumonia-due-tothe-novel-coronavirus-2019-ncovpress-briefing-transcript-30012020. pdf?sfvrsn=c9463ac1_2

6. WHO. Director General's opening remarks at the media briefing on COVID-19 [Internet]. [cited 05 October 2021]. Available from: https://www.who.int/docs/defaultsource/coronaviruse/transcripts/ 
who-audio-emergencies-coronaviruspress-conference-full-and-final11 mar2020.pdf?sfvrsn $=$ cb432bb3_2 [cited 05 October 2021].

7. Centers for Disease Control and Prevention. Situation summary [Internet]. [cited 05 October 2021]. Available from: https://covid.cdc.gov/ covid-data-tracker/\#datatracker-home

8. Centers for Disease Control and Prevention. How coronavirus spreads [Internet]. [cited 05 October 2021]. Available from: https://www.cdc.gov/ coronavirus/2019-ncov/prevent-gettingsick/how-covid-spreads.html

9. Centers for Disease Control and Prevention. Long-term effects of COVID-19 [Internet]. [cited 05 October 2021]. Available from: https://www.cdc. gov/coronavirus/2019-ncov/long-termeffects.html

10. Centers for Disease Control and Prevention. Telehealth [Internet]. [cited 05 October 2021]. https://www.cdc.gov/ coronavirus/2019-ncov/hcp/telehealth.html

11. American College of Obstetricians and Gynecologists. Committee Opinion Number 798. Implementing telehealth in practice. Obstet Gynecol [Internet]. [cited 05 October 2021];2020;135(2):e73-9. Available from: https://www.acog.org/ clinical/clinical-guidance/committeeopinion/articles/2020/02/implementingtelehealth-in-practice

12. Alverson DC, Holtz B, D'Iorio J, DeVany M, Simmons S, Poropatich RK. One size doesn't fit all: bringing telehealth services to special populations. Telemedicine and e-Health. 2008;14(9):957-63. https://doi. org/10.1037/a0036811

13. Dosaj A, Thiyagarajan D, Ter Haar $\mathrm{C}$, et al. Rapid implementation of telehealth services during the COVID19 pandemic. Telemed e-Health. 2021;27(2):116-20. https://doi. org/10.1089/tmj.2020.0219
14. Fryer K, Delgado A, Foti T, Reid $\mathrm{CN}$, Marshall J. Implementation of obstetric telehealth during COVID19 and beyond. Matern Child Health J. 2020;24(9):1104-10. https://doi. org/10.1007/s10995-020-02967-7

15. Zork NM, Aubey J, Yates H. Conversion and optimization of telehealth in obstetric care during the COVID19 pandemic. Semin Perinatol. 2020;44(6):151300. https://doi. org/10.1016/j.semperi.2020.151300

16. Krenitsky NM, Spiegelman J, Sutton D, Syeda S, Moroz L. Primed for a pandemic: implementation of telehealth outpatient monitoring for women with mild COVID-19. Semin Perinatol. 2020;44(7):151285. https://doi. org/10.1016/j.semperi.2020.151285

17. Madden N, Emeruwa UN, Friedman AM, et al. Telehealth uptake into prenatal care and provider attitudes during the COVID-19 pandemic in New York City: a quantitative and qualitative analysis. Am J Perinatol. 2020;37(10):1005. https://doi.org/10.1055/s-0040-1712939

18. Aziz A, Zork N, Aubey JJ, et al. Telehealth for high-risk pregnancies in the setting of the COVID-19 pandemic. Am J Perinatol. 2020;37(8):800-08. https://doi.org/10.1055/s-0040-1712121

19. Nouri SS, Khoong EC, Lyles CR, Karliner LS. Addressing equity in telemedicine for chronic disease management during the Covid-19 pandemic. NEJM Catalyst. 2020. https://catalyst.nejm.org/doi/ full/10.1056/CAT.20.0123

20. Bakhtiar M, Elbuluk N, Lipoff JB. The digital divide: how Covid-19's telemedicine expansion could exacerbate disparities. J Am Acad Dermatol. 2020;83(5):e345-6. https://doi. org/10.1016/j.jaad.2020.07.043

21. Ramsetty A, Adams C. Impact of the digital divide in the age of COVID-19. $J$ Am Med Inform Assoc. 2020;27(7):1147-8. https://doi.org/10.1093/jamia/ocaa078 
22. Ferguson JM, Jacobs J, Yefimova M, Greene L, Heyworth L, Zulman DM. Virtual care expansion in the Veterans Health Administration during the COVID-19 pandemic: clinical services and patient characteristics associated with utilization. $J$ Am Med Inform Assoc. 2021;28(3):453-62. https://doi. org/10.1093/jamia/ocaa284

23. Nemours KidsHealth (2018). What's a 'high-risk' pregnancy? [Internet]. [cited 05 October 2021]. Available from: https:// kidshealth.org/en/parents/high-risk.html

24. National Center for Health Statistics (NCHS) (2017). Urban-rural classification scheme for counties [Internet]. [cited 05 October 2021]. Available from: https://www.cdc.gov/ nchs/data_access/urban_rural.htm

25. Rodriguez JA, Saadi A, Schwamm LH, Bates DW, Samal L. Disparities in telehealth use among California patients with limited English proficiency.
Health Aff (Millwood). 2021;40(3):

487-95. https://doi.org/10.1377/ hlthaff.2020.00823

26. Centers for Medicare \& Medicaid Services. Medicaid [Internet]. [cited 05 October 2021]. Available from: https:// www.medicaid.gov/medicaid/index.html

27. Centers for Medicare \& Medicaid Services. Telemedicine [Internet]. [cited 05 October 2021]. Available from: https://www.medicaid.gov/medicaid/ benefits/telemedicine/index.html

Copyright Ownership: This is an open access article distributed in accordance with the Creative Commons Attribution Non Commercial (CC BY-NC 4.0) license, which permits others to distribute, adapt, enhance this work noncommercially, and license their derivative works on different terms, provided the original work is properly cited and the use is non-commercial. See: http://creativecommons.org/licenses/by-nc/4.0. 